\title{
Punktsieg für die Screening-Koloskopie
}

\author{
Endoskopische Darmkrebsvor- \\ sorgeuntersuchungen reduzieren \\ das Risiko für fortgeschrittene \\ Karzinome signifikant.
}

— Es gab lange Zeit keine randomisierte Studie, die eindeutig belegt, dass die Vorsorgekoloskopie das Risiko eines kolorektalen Karzinoms (CRC) und insbesondere die entsprechende Mortalität signifikant verringert. In einer Fall-Kontroll-Studie untersuchte man 1039 Patienten, die bei einer US-amerikanischen Krankenkasse versichert waren und zwischen 2006 und 2008 an einem kolorektalen Karzinom operiert wurden.

Eine Analyse der Daten von 471 auswertbaren Fall-Patienten und ihren 509 Kontrollen ergab, dass 13 Fall- $(2,8 \%)$ und 46 Kontrollpatienten $(9,0 \%)$ sich einer Vorsorgekoloskopie unterzogen hatten. Aus den Zahlen errechnet sich für diese Verfahren eine Odds ratio von 0,50 für alle kolorektalen Karzinome, von 0,79 für ein rechtsseitiges Spätkarzi-

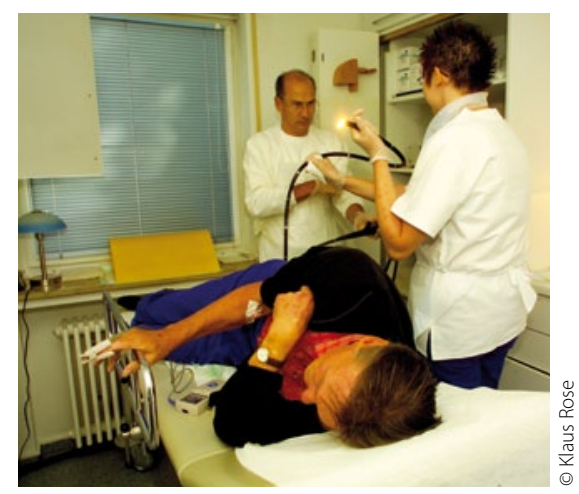

Der Wert der Vorsorge-Koloskopie ist jetzt auch wissenschaftlich belegt.

nom und von 0,26 für ein linksseitiges kolorektales Karzinom.

\section{- C. A. Doubeni et al.}

Screening colonoscopy and risk for incident late-stage colorectal cancer diagnosis in average-risk adults Ann. Int. Med. 2013; 158: $312-320$

\section{Kommentar}

Die Zahlen entsprechen in etwa denen einer Fall-Kontroll-Studie aus Deutschland, die im Jahr 2011 publiziert wurde, - aber eben aus Deutschland stammt und daher in der angelsächsischen Welt der Wissenschaft mit einem gewissen Hautgout belastet ist. Im Gegensatz zur Screening-Sigmoidoskopie weist die Vorsorgekoloskopie auch eine signifikante Verringerung der Rate fortgeschrittener rechtsseitiger Kolonkarzinome auf. Den Kritikern der Vorsorgekoloskopie wird allmählich der Wind aus den Segeln genommen. An der Tatsache, dass sie die Morbidität und Mortalität am CRC verringern kann, gibt es keinen Zweifel. Nun bleibt nur noch die Frage, ob das derzeitige Verfahren auch ökonomisch effizient ist und wie man z. B. durch die Vorschaltung besserer nicht invasiver Tests die Rate lediglich diagnostischer Koloskopien verringern und gleichzeitig den Anteil interventioneller Endoskopien erhöhen kann.

H. S. FÜESSL =

\section{Kein erhöhtes Krebsrisiko mit Insulinanaloga}

\begin{abstract}
In den vergangenen Jahren diskutierte man, ob Insulinanaloga, vor allem Insulinglargin, das Krebsrisiko im Vergleich zur Humaninsulintherapie erhöhen, da Glargin eine höhere Rezeptoraffinität und eine höhere mitogene Potenz als Humaninsulin aufweist.
\end{abstract}

— Nun wurden Daten aus dem französischen nationalen Gesundheitsversicherungssystem in Verbindung mit dem Krankenhausentlassungsprogramm in Frankreich ausgewertet. Erfasst wurden 70027 Patienten im Alter zwischen 40 und 79 Jahren, die von 2007 bis 2009 mit einer Basalinsulin-Therapie begonnen hatten.

$47432(67,7 \%)$ nahmen allein Insulinglargin ein, 12506 (17,9\%) wurden nur mit Insulindetemir behandelt, 4564
(6,5\%) erhielten nur basales Humaninsulin (BHI) und 5525 (7,9\%) zwei verschiedene lang oder mittellang wirksame Insuline.

Die Gesamtmortalitätsrate war in der BHI-Gruppe $(8,9 \%)$ höher als bei Insulinglargin- und Insulindetemir-Nutzern $(4,7 \%$ und $3,5 \%, p<0,0001)$. Die absolute Krebsereignisrate bei allen $\mathrm{Pa}$ tienten mit Glargin lag bei 1622/100 000 Personenjahren im Vergleich zu $1643 / 100000$ bei BHI. Auch für die Brustkrebsinzidenz ergab sich kein Unterschied zwischen den Glargin-Nutzern und Nutzern anderer Basalinsuline.

\footnotetext{
- J. P. Fagot et al.

Does Insulin Glargine Increase the Risk of Cancer Compared With Other Basal Insulins? A French nationwide cohort study based on national administrative databases. Diabetes Care 2013; 36(2): 294-301
}

\section{Kommentar}

Diese Studie unterstreicht die Ergebnisse der 2012 publizierten ORIGIN-Studie (N Engl J Med 11, June 2012) zur Sicherheit der Insulinglargin-Therapie. Bei einem medianen Follow-up von 6,2 Jahren hatten sich bei mehr als 12000 Patienten, die mit Insulinglargin behandelt wurden, im Vergleich zu einer Standard-Diabetestherapie ohne Insulinglargin kein erhöhtes Krebsrisiko gefunden. Die vorliegende Studie imponiert mit einer noch weit größeren Anzahl an Teilnehmern. Gespannt darf man auf die Folgeuntersuchungen sein, insbesondere was das Brustkrebsrisiko betrifft. Auch der Abfall des allgemeinen Krebsrisikos unter der Therapie mit Metformin und Sulfonylharnstoffen im weiteren Verlauf wird interessant.

C. JAURSCH-HANCKE - 